\title{
O PROGRAMA FARMÁCIA POPULAR: ALCANCE E LIMITES COMO ALTERNATIVA DE ACESSO A MEDICAMENTOS ESSENCIAIS
}

\author{
THE POPULAR PHARMACY PROGRAM: SCOPE AND LIMITATIONS AS AN ACCESS ALTERNATIVE TO ESSENTIAL \\ MEDICINES
EL PROGRAMA FARMAGIA POPULAR: ALCANCE Y LÍMITES COMO ALTERNATIVA DE ACCESO A MEDICAMENTOS ESENCIALES

\section{RESUMO}

Tendo como premissa que 0 Estado brasileiro reconhece 0 direito à saúde como uma das garantias de cidadania, 0 presente artigo analisa o Programa Farmácia Popular do Brasil, implantado em 2004, como política pública de acesso a medicamentos. Avaliou-se 0 programa com base em pesquisa documental, bibliográfica e entrevistas com atores-chave, para identificar seus alcances e limites. Ao objetivar a distribuição de medicamentos essenciais para o tratamento de doenças crônicas não transmissíveis, o programa apresentou algumas vantagens na eficiência logística, garantindo a disponibilidade de medicamentos, facilidade de acesso às farmácias conveniadas, mas denotou que os usuários do sistema público de saúde, que deveriam estar utilizando a assistência farmacêutica pública convencional, que distribui gratuitamente remédios à população, necessitavam de outra forma de atendimento, por causa das deficiências nessa assistência pública. Embora o programa tenha sido considerado importante como alternativa, foram sugeridas melhorias operacionais e estratégicas para que este se torne mais efetivo no acesso aos remédios pela população brasileira.

PALAVRAS-CHAVE: Programa Farmácia Popular, política pública, política social, acesso a medicamentos, gestão pública.

Silvia Rebouças Pereira de Almeida silvia_almeida7@hotmail.com

Professora da Universidade Presbiteriana Mackenzie, Faculdade de Computação e Informática - São Paulo - SP, Brasil

Maura Pardini Bicudo Véras mauraveras9@gmail.com

Professora da Pontifícia Universidade Católica de São Paulo, Programa de Pós-Graduação em Ciências Sociais - São Paulo - SP, Brasil

Submetido 03.11.2016. Aprovado 24.07.2017

Avaliado pelo processo de double blind review.

DOI: http://dx.doi.org/10.12660/cgpc.v22n72.64639 


\section{Abstract}

Based on the premise that the Brazilian state recognizes the right to health care as a citizenship guarantee, the present article analyses the Brazilian Popular Pharmacy Program, implemented in 2004, as a public policy for access to medicines. The program was analyzed by means of documentary and bibliographic research, as well as interviews conducted with program stakeholders, in order to identify its scope and limitations. Aiming at the distribution of essential medicines for the treatment of non-communicable chronic diseases, the program showed advantages regarding its efficient logistics, which ensures medicine availability and easy access to contracted private pharmacies. However, we found that public healthcare system users, who should be using conventional public pharmaceutical assistance, which distributes free medicines to the population, needed another form of assistance, due to the deficiencies of that service. Although the program was considered important as an alternative, operational and strategic improvements are suggested to make it more effective in providing access to medicines for the Brazilian population.

Keywords: Popular Pharmacy Program, public policy, social policy, access to medicines, public management.

\section{Resumen}

Con la premisa de que el Estado brasileño reconoce el derecho a la salud como una de las garantías de la ciudadan ía, este artículo analiza el Programa Farmacia Popular de Brasil, establecido en 2004 como una política pública de acceso a medicamentos. Se evaluó el Programa, con base en investigación documental, bibliográfica y entrevistas con actores clave, para identificar sus alcances y límites. Al analizar la distribución de medicamentos esenciales para el tratamiento de enfermedades crónicas no tranșmisibles, el Programa presentó algunas ventajas en la eficiencia log ística, lo que garantiza la disponibilidad de medicamentos y la facilidad de acceso a las farmacias conveniadas, pero denotó que los usuarios del sistema público de salud, que deberían utilizar la asistencia farmacéutica pública convencional que distribuye gratuitamente remedios a la población, necesitaban otra forma de atención debido a las deficiencias de dicha asistencia. Aunque el Programa se consideró importante como alternativa, se sugirieron mejoras operacionales y estratégicas para que sea más efectivo en lo que se refiere al acceso de la población brasileña a los medicamentos.

Palabras-clave: Programa Farmacia Popular, políticas públicas, política social, acceso a medicamentos, gestión pública.

\section{INTRODUÇÃO}

A Constituição Federal Brasileira de 1988 afirma que é dever do Estado a garantia do direito universal à saúde, e o uso de medicamentos é um instrumento necessário para promover a saúde coletiva. Entretanto, as políticas públicas voltadas a melhorar o acesso aos medicamentos no Brasil ainda necessitam ser aperfeiçoadas, para alcance mais efetivo, e requerem lentes mais finas em sua avaliação. Em sociedades marcadas por intensa desigualdade social como a nossa, avaliar uma política social reveste-se de grande significado. Se no período presentemente analisado já se notavam problemas nos programas voltados ao acesso a medicamentos, o quadro brasileiro se agravou a partir de maio de 2016, quando, no contexto da crise econômica e política, vimos assistindo a um verdadeiro desmonte das políticas sociais, a pretexto de contenção dos gastos públicos. Em especial, foram congeladas por 20 anos as despesas com saúde e educação, o que, sem dúvida, acentuará as deficiências já conhecidas do débito social brasileiro.

Já em 2001, a Organização Mundial da Saúde afirmava que pelo menos um terço da população mundial não tinha acesso adequado a medicamentos essenciais (World Health Organization, 2001) e, de acordo com estudo desenvolvido pela Gazeta Mercantil (2000), essa proporção pode chegar a $40 \%$ da população brasileira, devido a preços elevados, 
sendo a única alternativa o fornecimento gratuito pelo Sistema Único de Saúde (SUS). Apenas as famílias com maior poder aquisitivo têm condições de obtê-los, dados os descontos concedidos pelas farmácias, planos privados de saúde e de assistência farmacêutica, programas de fidelização de clientes dos laboratórios farmacêuticos e, ainda, a alternativa dos medicamentos genéricos, mais baratos que os de referência.

Desde 1988, o conceito de saúde foi estabelecido como direito universal, ampliado e vinculado às políticas sociais e econômicas, como dever constitucional de todos os níveis governamentais. A assistência passou a ser concebida de modo integral, ou seja, preventiva e curativa. A Lei n. 8.080/90 consolidou as orientações constitucionais do SUS e a Lei n. 8.142/90 tratou do envolvimento da sociedade nas questões da saúde, criando conferências e conselhos em cada nível de governo. Enfim, o direito à saúde foi positivado como um direito social, abrangendo a assistência farmacêutica. Pela Lei Orgânica da Saúde, as assistências terapêuticas e farmacêuticas devem ser garantidas integralmente aos cidadãos brasileiros, exigindo, pois, políticas, serviços de saúde e assistência farmacêutica em escala coletiva. No SUS, a gestão do sistema é realizada pelas três esferas de governo, municipal, estadual e federal, compartilhando responsabilidades e financiamento. Os serviços de saúde são principalmente administrados pelos municípios, sob a gestão dos estados e da União.

Pela Constituição Brasileira de 1988, com a criação do SUS, a assistência farmacêutica passou a ser responsabilidade do setor público, como alternativa de acesso a medicamentos de modo complementar ao aten- dimento de saúde feito pelas unidades básicas do SUS (Ministério da Saúde, 2013). O SUS é um sistema de políticas públicas de saúde que movimenta uma rede de organizações, programas e estratégias, visando a garantia da saúde, e, desde a sua criação, em 1988, representa o principal canal de acesso público da população brasileira aos medicamentos.

No que tange ao mercado global de medicamentos, seu valor é estimado em mais de 600 bilhões de dólares anuais, e os custos com medicamentos em países não desenvolvidos alcançam $50 \%$ do total gasto com saúde (World Health Organization, 2006). De acordo com essa mesma fonte, no período de 1985 a 1999, o valor mundial de produção de medicamentos cresceu quatro vezes mais rapidamente que o produto interno bruto global, dominado por um pequeno grupo de grandes multinacionais, e dois terços dessa produção estão concentrados em cinco países: Estados Unidos, Japão, Alemanha, França e Reino Unido (World Health Organization, 2004).

O acesso aos medicamentos esbarra em questão estrutural da indústria farmacêutica, pois a estratégia competitiva do setor baseia-se na diferenciação de produtos e, principalmente, na publicidade para conquistar a imagem de melhor qualidade na percepção da classe médica. Nos últimos anos, o volume de recursos em publicidade representa em torno de $30 \%$ dos preços dos medicamentos, enquanto os gastos em Pesquisa \& Desenvolvimento (P\&D) representam cerca de 12 a $15 \%$ do preço final (Picarelli \& Aranha, 2001).

O setor farmacêutico apresenta crescente 
concentração de indústrias de grande porte, em sua maioria multinacionais, que têm o monopólio de mercado para medicamentos de referência por 20 anos, garantido pelo regime de patentes. Isso Ihes proporciona maior poder na determinação dos preços, geralmente elevados e, portanto, impraticáveis para a população. Esse cenário de alta complexidade deve ser especialmente considerado por países que possuem sistemas universais de saúde, como é o caso do Brasil, que inclui a garantia de acesso a medicamentos como parte da sua política de saúde (Amorim \& Perillo, 2008). A regulamentação dos medicamentos genéricos no Brasil, implementada em 1999, foi uma tentativa de facilitar o acesso da população a eles, por apresentarem preços em geral em torno de $40 \%$ mais baratos que os remédios de referência, permitindo melhor aproveitamento da verba pública.

Dois pontos a destacar: 1) a necessidade de revisão da carga tributária para a produção dos medicamentos, que são bens essenciais; de acordo com o Sindicato da Indústria de Produtos Farmacêuticos no Estado de São Paulo (Sindusfarma, 2014), em 2014 , esse imposto girava em torno de $33 \%$ sobre o preço, e a diminuição dos tributos o reduziria; e 2) a perversa tendência de governos liberais não assumirem prioridade nas políticas de saúde e reduzirem seus gastos com medicamentos, devido aos escassos recursos disponíveis para o setor, embora as necessidades com a saúde sejam crescentes ao longo dos anos (World Health Organization, 2001). Em 2006, a despesa da União com medicamentos foi de $R \$ 4,3$ bilhões, ou $10,6 \%$ do gasto federal em saúde. De acordo com o Ministério da Saúde (2015), esse gasto vem sendo tri- plicado, somando $\mathrm{R} \$ 12,9$ bilhões em 2014 , representando em torno de $14,0 \%$ dos $R \$$ 92,2 bilhões destinados ao custeio de todo o SUS. Em 2015, estimou-se a compra de remédios em R\$14,3 bilhões do Governo Federal (Caderno de Economia \& Negócios, 2015, p. H5).

A política pública de medicamentos e assistência farmacêutica, estabelecendo critérios de inclusão e exclusão de remédios, teve um período de transição durante os anos de 2000 a 2004, com diversos atos administrativos, e, posteriormente, adequou-se aos compromissos sociais do Governo Lula, declarados na direção do atendimento às camadas menos privilegiadas.

Embora reconhecendo a complexidade envolvida no acesso aos medicamentos, o presente estudo limitou seu objetivo principal em realizar uma análise crítica do alcance e limites da política pública do Programa Farmácia Popular, proposta em 13 de abril de 2004 pelo Governo Federal, com a Lei $n$. 10.858, no primeiro mandato do Presidente Luiz Inácio Lula da Silva. Restringiu-se essa análise a um levantamento de dados junto aos atores-chave na cidade de São Paulo.

A avaliação de uma política social envolve muitas facetas, sem que se consiga desenvolvê-las todas nos limites deste trabalho. Mas importa resgatar que devem ser vistas em sua forma contraditória, pois são políticas de Estado, e, se visam atender grupos integrantes das esferas de poder de diferentes frações do capital, em especial, o financeiro, industrial e comercial, também devem observar as pressões das camadas populares, dos trabalhadores e outras forças sociais (Faleiros, 2009). Nesse contexto, as contradições 
inerentes ao sistema capitalista permeiam o desenvolvimento das políticas públicas de saúde, em geral, e, em particular, as de acesso a medicamentos. O Programa Farmácia Popular, se considerado uma política social de saúde do Estado, pode constituir estratégia de redistribuição de renda, fazendo referência ao princípio da universalidade com equidade (Boschetti, Behring, Santos, \& Mioto, 2010). A política social, identificada como uma política de ação, é uma espécie de política pública, e inclui todas as políticas que requerem a participação ativa do Estado, sob o controle da sociedade, no planejamento e execução de procedimentos e metas voltados para a satisfação de necessidades sociais. Assim, tem perfil, funções e objetivos próprios e produz impactos no contexto em que atua. Assim, o Programa Farmácia Popular pode ser identificado como uma política de ação, produzindo impactos no contexto em que atua, exercendo influência no acesso efetivo à saúde, pelo tratamento medicamentoso (Pereira, 2008).

Ao ampliar o acesso aos medicamentos para determinadas doenças, com prioridade às crônicas não transmissíveis, ele proporciona maior adesão aos tratamentos prescritos e oferece maior bem-estar aos cidadãos, objetivos de políticas sociais. Busca-se, pois, avaliar o programa como alternativa de acesso aos medicamentos pela população e em sua importância aos participantes. Por meio de 17 entrevistas com os atores-chave, identificaram-se oportunidades de melhorias estratégicas e operacionais para que possa se tornar mais efetivo nos seus fins precípuos e mais atrativo aos diversos participantes, incluindo indústrias farmacêuticas, fornecedoras no programa, e farmácias particulares credenciadas.
Comentando brevemente informações metodológicas, baseado em pesquisa de doutorado sobre alcances e limites do programa, o estudo combinou procedimentos de compilação documental e fontes secundárias, estatísticas em saúde e ampla pesquisa bibliográfica sobre o setor farmacêutico, políticas sociais e programas de governo para o acesso aos medicamentos, com foco no Programa Farmácia Popular. Em segunda etapa, foram realizadas, para uma pesquisa qualitativa, 17 entrevistas em profundidade, junto aos principais segmentos do setor participantes do programa, no âmbito tanto empresarial quanto público do setor farmacêutico, abordando posições de liderança nesses segmentos. Embora a sua abrangência seja nacional, foi selecionada a cidade de São Paulo no levantamento dos atores-chave do programa, sendo esta a maior cidade do País, com importante estrutura na área de saúde e de fácil acesso na coleta de informações.

Assim, foram realizadas as seguintes entrevistas:

- três redes de farmácias particulares credenciadas, priorizando os funcionários com posições de liderança, responsáveis pela gestão, regulamentação e operacionalização do programa;

- representantes de duas indústrias farmacêuticas multinacionais fabricantes de medicamentos de referência, consultadas as gerências responsáveis por assuntos relacionados ao governo, incluindo o Programa Farmácia Popular. Uma dessas empresas colaborou na fase de coleta de campo como facilitadora, informando os responsáveis pelo Programa Farmácia Popular no Minis- 
tério da Saúde, em Brasília;

- o gerente de Marketing de uma indústria farmacêutica multinacional, fabricante de medicamentos genéricos, com participação importante no programa;

- entrevista junto ao órgão do Estado responsável pela gestão do programa, o Ministério da Saúde, em Brasília, feita presencialmente com o coordenador-geral de gestão (CGG) do programa, e as informações estatísticas foram obtidas no site do Ministério da Saúde. Foi também consultada a coordenadora do programa nos anos de 2006 e 2007, quando este teve uma mudança estratégica: a integração do Governo Federal com as farmácias particulares;

- três especialistas das áreas de Economia, Administração e Serviço Social da Pontifícia Universidade Católica de São Paulo (PUC-SP), com estudos desenvolvidos em saúde pública e medicina social;

- três entidades de classe no segmento farmacêutico, como o Sindusfarma, sendo consultado o seu presidente executivo, a Associação Brasileira de Rede de Farmácias e Drogarias (Abrafarma), também com seu presidente executivo, e a Associação Brasileira das Indústrias de Medicamentos Genéricos, a Pró Genéricos, sendo consultado o gerente técnico administrativo;

- três entrevistas na Secretaria Municipal de Saúde de São Paulo para a obtenção de informações sobre a assistência farmacêutica prestada nas unidades do SUS: foram entrevistados o Secretário-Geral da Secretaria Municipal da Saúde; a coordenadora da Área Técnica de Assistência Farmacêutica e o diretor da Divisão Técnica de Suprimentos;

- alguns conselheiros de Saúde e usuários do programa também contribuíram com informações sobre a satisfação dos pacientes usuários do SUS e do Programa Farmácia Popular.

Buscando revelar alcance e limites do Programa Farmácia Popular, a presente análise crítica se estrutura nos seguintes aspectos: exposição dos objetivos e organização institucional do programa, suas alterações e desenvolvimento ao longo de diferentes conjunturas, e seu desempenho como política pública, evidenciado pelos volumes de recursos públicos a ele destinados, pela lista de fármacos selecionada e pelo envolvimento da rede de estabelecimentos conveniados.

\section{ANÁLISE DO PROGRAMA FARMÁCIA PO- PULAR}

\section{Objetivos e organização institucional}

O programa tem como objetivo ampliar o acesso aos medicamentos essenciais, com prioridade aos tratamentos de doenças crônicas não transmissíveis, como diabetes, hipertensão, asma, colesterol, mal de Parkinson e osteoporose. Pretende estender a aquisição dos medicamentos e insumos essenciais a baixo custo, com descontos especiais de até $90 \%$ nos preços, ou gratuitamente, ampliando o acesso a mais lugares e pessoas, ao aproveitar a dinâmica de toda a cadeia farmacêutica, incluindo no sistema as indústrias farmacêuticas, distribuidores, farmácias e pacientes, seus usuários. Em conexão com a implantação do SUS, considerado o principal canal de acesso público a medicamentos, o Programa Farmácia Popular contribuiria para um alívio nas despesas familiares, especialmente às classes populares, que, mesmo usuárias do sistema privado de saúde, apresentam dificuldades para adquirir medicamentos no mercado regular. 
Outro objetivo do programa é qualificar a assistência farmacêutica, oferecendo, além dos medicamentos, o atendimento pelos farmacêuticos nas farmácias conveniadas.

Inicialmente, o programa contou com uma rede própria de Farmácias Populares implementadas pelo Governo Federal, sob a gestão da Fundação Oswaldo Cruz (Fiocruz); a partir de 2004, estendeu-se para as Farmácias Populares em parceria com os estados e municípios. De acordo com a Federação Brasileira da Indústria Farmacêutica (Febrafarma, 2008), o programa, na concepção original, era uma ação direta do Governo Federal na escolha, produção, distribuição e venda subsidiada de medicamentos. A escolha inicial de tais fármacos foi feita com prioridade aos tratamentos de diabetes e hipertensão. Os laboratórios estatais produziriam os remédios e os distribuiriam às farmácias próprias do Governo Federal e em parceria com os estados e municípios. A proposta de fornecimento de medicamentos a baixo custo pelo Laboratório Farmacêutico do Estado de Pernambuco (Lafepe), desde 2001, serviu de modelo para essa nova iniciativa federal (Santos-Pinto, Costa, \& Osorio-de-Castro, 2011).

Diferindo do modelo adotado pelo SUS na distribuição gratuita de todos os remédios prescritos, o programa adotou um novo instrumento de ação na política de medicamentos, que é o copagamento, em que o paciente recebe o remédio pretendido com um subsídio governamental, mas também paga a diferença no preço. E, quanto à questão crítica do SUS, em que estoques localizados em postos e hospitais específicos são de acesso limitado, ou nas grandes cidades, onde pontos de distribuição são, muitas ve- zes, localizados em regiões distantes, implicando custo extra de transporte, houve a vantagem do programa pela maior capilaridade geográfica no atendimento das Farmácias Populares.

Contudo, no final de 2006, o governo lançou nova modalidade para a distribuição de medicamentos, com a possibilidade de credenciamento da rede privada de farmácias no Programa "Aqui Tem Farmácia Popular." As mudanças se devem a um conjunto de fatores, nos quais a rede pública, com farmácias próprias do governo e em parceria com estados e municípios, mostrou-se limitada para um crescimento mais expressivo pelas regiões do País devido, principalmente, aos custos elevados de implantação e manutenção. Uma vantagem importante foi a mudança estrutural do novo modelo de distribuição, com a integração das farmácias privadas, pois, na rede pública, o programa opera com custos fixos e operacionais muito altos, além do subsídio aos medicamentos implícitos nos preços, enquanto, na nova forma de integração com as farmácias particulares, esses custos desaparecem, uma vez que toda a transação é absorvida pela operação regular, utilizando as instalações, pessoal, tecnologia, gestão de estoques e logística empresarial. Nesse novo molde, os medicamentos do Programa Farmácia Popular são apenas alguns daqueles adicionais entre os normalmente ali comercializados. A gestão logística eficiente das farmácias particulares confere outra vantagem a essa forma de distribuição de medicamentos à população, pois garante sua disponibilidade na quantidade e momento necessários para adesão aos tratamentos prescritos.

\section{Alterações e desenvolvimento}


O governo lançou, em 14 de fevereiro de 2011, o Programa "Saúde não Tem Preço", estabelecendo gratuidade nos tratamentos considerados prioritários, como diabetes e hipertensão e, a partir de junho de 2012, para o tratamento de asma. Nesse programa, as farmácias passam a ser ressarcidas pelo Ministério da Saúde no valor integral dos medicamentos, de acordo com o valor de referência estabelecido pelo Governo Federal (Febrafarma, 2008).

Segundo o coordenador do programa no Ministério da Saúde (2013), a meta federal era alcançar 600 farmácias do governo no País, que distribuíssem 113 itens, mediante processo de licitação nas compras de medicamentos; nas farmácias particulares credenciadas ao "Aqui tem Farmácia Popular," são distribuídos 24 itens, definidos pelo Ministério da Saúde, ficando a critério dessas farmácias a escolha dos fornecedores. A indústria farmacêutica pública atende apenas à demanda de medicamentos adquiridos diretamente pelo governo, mediante licitação de compras, e esse fornecimento não se estende às redes privadas.

De acordo com a Fiocruz (2014), gestora das farmácias do governo e em parceria com os estados e municípios, em São Paulo, capital, a proporção das receitas oriundas do sistema de saúde particular soma aproximadamente $59,7 \%$ do total, enquanto os 40,3\% restantes são provenientes do SUS e de entidades filantrópicas, como a Santa Casa de Misericórdia. Mesmo que esses dados representem apenas uma amostra das receitas encaminhadas nas farmácias do governo, já que são compiladas manualmente por funcionários, processo sujeito a falhas, essa porcentagem representa uma distorção na proposta inicial do programa, e os elevados percentuais das receitas procedentes do SUS sinalizam deficiências na assistência farmacêutica ali praticada, as quais comprometem a disponibilidade aos pacientes, que acabam buscando as Farmácias Populares como cobertura a tais falhas, mesmo que tenham que pagar uma parcela do seu preço, no sistema de copagamento.

Um estudo realizado pela Controladoria-Geral da União (CGU), no período de agosto de 2004 a julho de 2006, em municípios de até 500 mil habitantes, afirmava que a assistência farmacêutica prestada pelo SUS vinha apresentando problemas graves e recorrentes de ineficiência logística, com falhas nos controles, fraudes e desvios de recursos, acabando por comprometer a disponibilidade de medicamentos aos potenciais beneficiários (Vieira, 2008). Tal pesquisa veio demonstrar que o principal entrave à provisão pública de medicamentos nas unidades básicas do SUS estava na sua má gestão, em termos de falhas em planejamento e controle na distribuição dos fármacos, mais do que na escassez dos recursos públicos a esse modelo de acesso.

\section{Desempenho da política pública}

A presente análise crítica foca como aspectos fundamentais: os valores públicos transferidos ao programa, o número de entidades conveniadas e a adequação da lista de remédios selecionada para operar.

Houve um aumento significativo do gasto público ao longo dos anos, em valores transferidos do Ministério da Saúde às farmácias particulares credenciadas ao "Aqui Tem Farmácia Popular,', passando de $\mathrm{R} \$ 34,7$ 
milhões em 2006, início da iniciativa, para um patamar de $\mathrm{R} \$ 2,4$ bilhões em 2014. A evolução da verba pública destinada ao pro- grama está apresentada na Tabela 1, e os gastos públicos com as farmácias do governo, na Tabela 2.

Tabela 1. Gastos do Ministério da Saúde no programa "Aqui tem Farmácia Popular"

\begin{tabular}{|c|c|}
\hline Ano & Valores em R\$ milhões \\
\hline 2006 & 34,7 \\
\hline 2007 & 157,1 \\
\hline 2008 & 323,8 \\
\hline 2009 & 287,6 \\
\hline 2010 & 247,2 \\
\hline 2011 & 831,1 \\
\hline 2012 & $1.397,6$ \\
\hline 2013 & $1.862,2$ \\
\hline 2014 & $2.411,8$ \\
\hline
\end{tabular}

Fonte: FNS/MS - Ministério da Saúde (2015).

Tabela 2. Gastos do Ministério da Saúde com farmácias próprias do governo

\begin{tabular}{|c|c|}
\hline Ano & Valores em R\$ milhões \\
\hline 2011 & 63,6 \\
\hline 2012 & 62,6 \\
\hline 2013 & 59,4 \\
\hline 2014 & 64,9 \\
\hline
\end{tabular}

Fonte: FNS/MS - Ministério da Saúde (2015).

São pouco expressivos os gastos públicos destinados às farmácias do governo, comparados aos investimentos no "Aqui Tem Farmácia Popular", refletindo a estratégia de crescimento baseada na integração com a rede privada de farmácias. Os gastos do Governo Federal com o "Aqui Tem Farmácia Popular" são também muito superiores àqueles da assistência farmacêutica nas unidades básicas do SUS, que totalizaram R $\$ 1,05$ bilhão em 2014 (Ministério da Saúde, 2015). Estudo recente estima que os custos do Programa Farmácia Popular, na modalidade de integração com a iniciativa privada, giram em torno de $150 \%$ a mais que os serviços de assistência farmacêutica nas unidades básicas do SUS (Carraro, 2014).

O Programa Farmácia Popular define sua lista de medicamentos baseado na Relação Nacional de Medicamentos Essenciais (Rename), utilizada como base para o rol de medicamentos essenciais das unidades básicas do SUS. A prioridade é dada ao atendimento às patologias crônicas não transmissíveis, sendo esses medicamentos eleitos para gratuidade, como é o caso dos tratamentos de diabetes, hipertensão e 
asma. Nessa definição, também foram considerados os medicamentos que compõem os programas assistenciais e de Secretarias do Ministério da Saúde. A maior parte, em torno de $80 \%$ dos medicamentos distribuídos, é de genéricos; entre $6 \%$ e $8 \%$ são remédios de referência; e cerca de $12 \%$ a $14 \%$ são similares (Ministério da Saúde, 2013).

Atores-chave do programa entrevistados sugeriram um catálogo mais amplo de medicamentos distribuídos, incluindo mais princípios ativos para patologias já atendidas, mais modernos e de melhor qualidade, e a inclusão na lista de outras classes de remédios, como antibióticos, analgésicos, anti-inflamatórios, medicamentos de alto custo e para o tratamento de doenças tropicais e endêmicas, mal de Alzheimer, depressão e outras doenças do sistema nervoso central.

De acordo com a sua coordenação no Ministério da Saúde (2013), o programa é considerado inovador pela capilaridade geográfica, e a meta é ampliar, por meio da integração com as redes de farmácias particulares. $O$ programa atende 21.951 .668 pacientes usuários (Associação Brasileira das Indústrias de Medicamentos Genéricos, 2014). Entretanto, como desafio para o crescimento, foi mencionada a existência de municípios sem farmácias e drogarias, municípios que dispõem destas, porém sem registros, ou sem acesso à internet para a comunicação com o Ministério da Saúde.

Gráfico 1. Evolução do número de farmácias próprias do governo

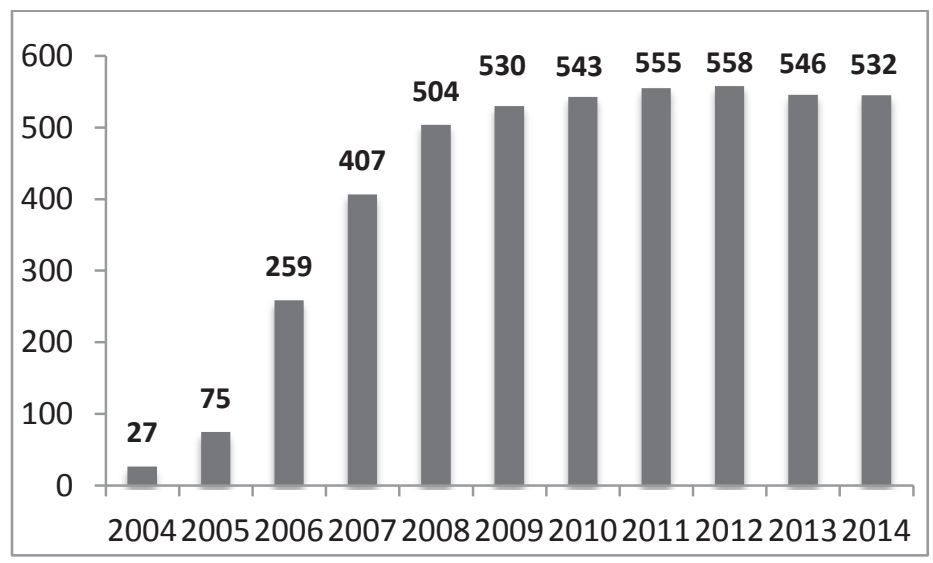

Fonte: DAF/SCTIE/MS - Ministério da Saúde (2015). 
Gráfico 2. Número de municípios atendidos pela rede própria do governo

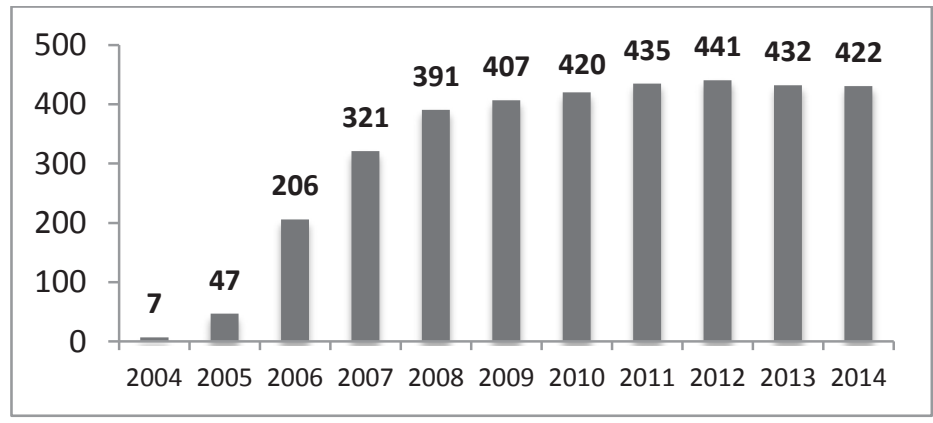

Fonte: DAF/SCTIE/MS - Ministério da Saúde (2015).

Observa-se estabilização e até encolhimen- modelos de rede própria do Governo Federal to no número de Farmácias Populares e mu- e em parceria com os estados e municípios. nicípios atendidos pelo programa dentro dos

Gráfico 3. Número de farmácias do programa "Aqui tem Farmácia Popular"

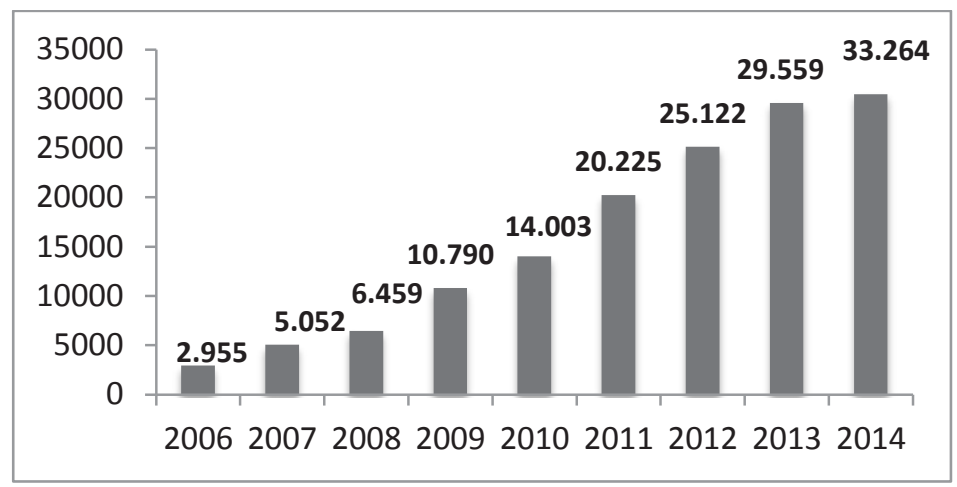

Fonte: DAF/SCTIE/MS - Ministério da Saúde (2015). 
Gráfico 4. Número de municípios atendidos pelo programa "Aqui tem Farmácia Popular"

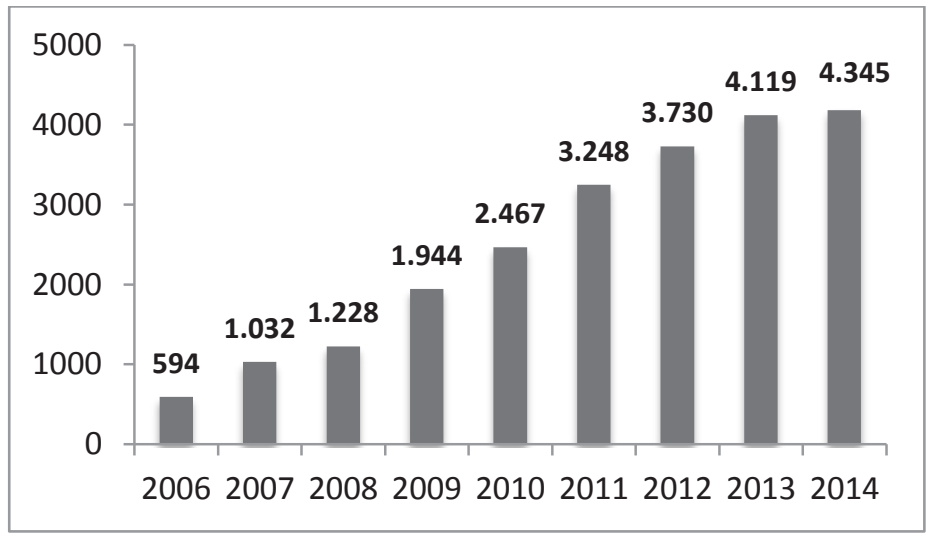

Fonte: DAF/SCTIE/MS - Ministério da Saúde (2015).

Os dados demonstram crescimento expressivo no número de estabelecimentos credenciados, aumentando de 2.955 pontos de venda em 2006 para 33.264 farmácias particulares em 2014. Houve também um aumento significativo no número de municípios atendidos, passando de 594, em 2006, para 4.345, em 2014 (Ministério da Saúde, 2015).

Entretanto, de acordo com a Abrafarma (2014), o programa apresenta limitações no atendimento aos municípios menores, nos quais estão presentes, em sua maioria, as farmácias de pequeno porte, que não têm as mesmas condições de negociação que as redes de farmácias junto aos fornecedores, dado o menor volume de medicamentos comercializados. Além disso, tais estabelecimentos de pequeno porte apresentam outras limitações - como baixo capital de giro para investimentos em estoques e tecnologia da informação - impactando negativamente na participação no programa.

As redes de farmácias localizadas nas gran- des cidades têm maior poder de barganha e conseguem negociar melhores descontos junto aos fornecedores, viabilizando a comercialização dos medicamentos no programa, especialmente aqueles oferecidos com gratuidade, considerando que é baixo o valor que lhes é ressarcido pelo governo. Essas redes também possuem maior capacidade para investimentos em tecnologia da informação para a gestão logística eficiente e para viabilizar a comunicação com o Ministério da Saúde.

Ainda no que se refere aos aspectos financeiros, nas palavras do presidente executivo do Sindicato da Indústria de Produtos Farmacêuticos no Estado de São Paulo (2013), que representa esse segmento industrial no estado,

os valores de referência estabelecidos pelo governo para o ressarcimento às farmácias particulares necessitam, pelo menos, cobrir os custos de fabricação, pois nenhuma indústria tem condições de vender medicamentos com prejuízo financeiro. Para a 
indústria farmacêutica não é interessante que seus produtos faltem nas farmácias credenciadas, pois, se a classe médica prescreve o produto e os pacientes não o encontram disponível, provavelmente, numa próxima vez, prescreverá outra marca.

Ele sugere que o modelo de copagamento seja adotado para todos os medicamentos no programa, a exemplo da Espanha, e comenta que os valores de referência necessitam ser reajustados, pois o governo estabeleceu, desde o início das operações, um valor médio para esses preços, que, desde então, não foi corrigido. $O$ sindicato aponta que a gratuidade estabelecida para alguns medicamentos somente foi alcançada graças a maiores descontos concedidos pela indústria farmacêutica. Foi sugerida prioridade ao atendimento das classes menos favorecidas, considerando que o Brasil não está no mesmo patamar de desenvolvimento econômico e social de países desenvolvidos, onde a assistência farmacêutica é universal, como é o caso da Inglaterra, referência em saúde pública.

Do ângulo das redes de varejo pesquisadas, com atuação na cidade de São Paulo, o Programa Farmácia Popular é considerado importante para essas empresas, pois, além de proporcionar rentabilidade financeira, aumenta o fluxo de consumidores nas lojas, que retiram os medicamentos no programa e acabam comprando também outros produtos. $O$ programa traz às lojas um novo segmento de mercado, constituído pela população das classes de menor renda, que passaram a ser novas consumidoras dessas farmácias.
No que se refere aos aspectos operacionais do programa, o Ministério da Saúde (2013) afirma que, para se obter o benefício, basta que o paciente apresente o seu número de Cadastro de Pessoa Física (CPF), documento com fotografia e a receita médica, com validade de 120 dias, contendo o número do CRM do médico, em qualquer um dos estabelecimentos credenciados ao programa "Aqui Tem Farmácia Popular" ou nas farmácias do governo. Esses dados são inseridos em softwares das farmácias e as informações são enviadas, via internet, para - Ministério da Saúde, para analisar e autorizar o benefício. Esse ponto é também crítico, pois o sistema de comunicação entre as lojas e o Ministério da Saúde tem falhas, trazendo impacto negativo nas retiradas dos remédios pelos pacientes e no ressarcimento às farmácias.

Apontou-se, como necessidade de melhoria, a ampliação dos mecanismos de segurança para evitar fraudes, realizando verificação das informações na autorização para o fornecimento dos medicamentos nas farmácias, comparando com as retiradas feitas nas unidades do SUS (Ministério da Saúde, 2013).

Os entrevistados das farmácias opinam que o programa poderia ser mais efetivo como alternativa de acesso aos medicamentos mediante a simplificação do processo de aprovação do benefício pelo Ministério da Saúde, apontado como muito burocrático. Foi também indicada a necessidade de o governo ampliar o período de credenciamento dos estabelecimentos, o qual ocorre apenas uma vez ao ano. Após o pedido de credenciamento, normalmente demora-se mais de um ano para que uma nova loja possa ope- 
rar no programa.

A mesma direção foi apontada em entrevista feita, em 2014, com a coordenadora do Programa Farmácia Popular (2014) nos anos de 2006 e 2007, observando a necessidade de se ampliarem os investimentos em tecnologia da informação nos programas governamentais de assistência farmacêutica, para a melhoria de controles, maior eficiência nas operações logísticas e em toda a cadeia de suprimentos de medicamentos à população.

Segundo especialistas, como Regina Marsiglia (2014), docente de Medicina Social e Serviço Social, o Programa Farmácia Popular representa mais uma ação do Governo Federal para tentar cobrir a deficiência no atendimento da assistência farmacêutica nas unidades do SUS, que fornece gratuitamente à população. A pesquisadora acredita que o programa foi criado com o objetivo de dar maior visibilidade ao Governo Federal perante a população e que a imagem de inovação não reflete a realidade, pois, antes dele, o governo já distribuía gratuitamente uma lista de medicamentos essenciais, baseada na Rename, nas unidades básicas do SUS. Além disso, mesmo antes da aprovação do SUS na Constituição Federal de 1988, já havia a Central de Medicamentos (CEME) no Ministério da Saúde, criada em 1971 com o objetivo de melhorar o acesso aos medicamentos por meio do sistema público de saúde, em que vários deles eram produzidos pela Fundação para o Remédio Popular (FURP). Em São Paulo, num outro exemplo, em 1995, a Secretaria de Estado da Saúde ampliou o número de medicamentos a serem distribuídos com o programa "Dose Certa".
Além desses programas apontados pela pesquisadora, o "Programa Remédio em Casa”, implementado em 2005 pela Secretaria Municipal da Saúde de São Paulo, tem tido muito sucesso como alternativa de acesso aos essenciais, incluindo a distribuição de 12 medicamentos, gratuitamente entregues pelo correio nos domicílios dos pacientes.

\section{CONSIDERAÇÕES FINAIS}

O Programa Farmácia Popular é uma iniciativa importante do Governo Federal para ampliar o acesso aos medicamentos para mais pessoas e regiões do Brasil, ao mesmo tempo que vem contribuir para aliviar as despesas familiares, principalmente as das camadas populares, em que os gastos com medicamentos têm maior peso no orçamento.

Uma breve reflexão sobre as características do Estado brasileiro pode auxiliar a entender os limites de uma política social como o programa em pauta. No Brasil, não houve um Estado do Bem-Estar Social como se apresentou aos europeus no período pós-Segunda Guerra, tendo mesmo sido chamado por alguns entre nós de "Estado do Mal-Estar Social" (Oliveira, 2004). O caso latino-americano, segundo esse autor, no entanto, acompanhou o desmonte mundial da proteção social ocorrida após 1970 com a chamada reestruturação produtiva e do capital, processos agravados na década de 1990, o que se radicalizou com a crise financeira de 2007/2008, com a austeridade fiscal. Essa rede anterior de proteção social abrangia o seguro-desemprego, assistência social, previdência e sistemas de saúde, ou seja, participava da construção do ambiente social e urbano. A partir das políticas neoliberais e 
do Estado mínimo, as ditas políticas sociais se encolheram e buscaram atender o essencial, sem assumir a universalidade das ações, ao contrário da implantação do SUS, este, sim, um programa de alcance universal. O Programa Farmácia Popular se insere nesse contexto.

Observou-se, ao longo dos anos, que uma parcela importante de usuários do Programa Farmácia Popular é proveniente das unidades de atendimento do SUS, o que revela ineficiência na assistência pública farmacêutica, a comprometer a disponibilidade dos medicamentos gratuitos à população. O direcionamento de usuários do SUS às Farmácias Populares representa, portanto, uma distorção no público-alvo do programa, além de ter beneficiado os consumidores de estratos médios da população, clientes das redes de farmácias populares, uma vez que o SUS tem servido mais às chamadas camadas populares.

A estratégia de crescimento do programa baseada na integração com a iniciativa privada foi considerada o melhor caminho para o seu crescimento, pois concentrou o direcionamento da verba pública no subsídio aos medicamentos, e não na implantação e manutenção de farmácias do governo, ao mesmo tempo que conta com a gestão logística eficiente das farmácias particulares, garantindo, assim, a disponibilidade dos medicamentos à população. Porém, é imprescindível que o Governo Federal reveja os valores de referência ressarcidos às farmácias e também estude a possibilidade de isenção ou redução de impostos sobre os medicamentos, que são considerados bens essenciais, a exemplo de outros países, como os Estados Unidos, Reino Unido, Ca- nadá, Suécia, México e Colômbia, em que os medicamentos são isentos de impostos (Sindusfarma, 2013).

Foi indicado, como limitação do Programa "Aqui Tem Farmácia Popular", o fato de as farmácias de pequeno porte encontrarem maior dificuldade para operarem, dado o baixo valor de referência ressarcido pelo governo, o que fragiliza ainda mais o acesso aos medicamentos nos municípios menores. A redução na burocracia se faz necessária, tanto no processo de renovação e cadastramento de novas farmácias particulares como na concessão do benefício aos pacientes pelo Ministério da Saúde. Novos investimentos em tecnologia da informação agilizariam esses processos por meio do uso da internet.

Poderia ser avaliada e mantida a prioridade a alguns segmentos sociais no programa, como os de baixa renda e, ainda, por exemplo, os públicos infantil e idoso, para a distribuição de medicamentos mais modernos. O programa deve ser notado como importante alternativa de acesso aos medicamentos, mas este não isenta o Estado do seu dever de garantir a sua disponibilidade gratuita nas unidades de atendimento do SUS, pois uma parcela importante da população ainda depende da gratuidade para o acesso aos medicamentos no Brasil.

Vale lembrar, ainda, que o programa "Aqui Tem Farmácia Popular", na modalidade de parceria com a iniciativa privada, por ser mais caro aos cofres públicos que o modelo de distribuição de fármacos convencional nas unidades básicas do SUS, esbarra numa premissa constitucional em que o Estado deve atentar para a questão da econo- 
micidade na alocação dos recursos públicos entre os programas de governo, e, até o momento, não foi comprovada efetividade superior na integração com a iniciativa privada que justifique essa prioridade, em detrimento da provisão pública de medicamentos à população pelo SUS.

O desenvolvimento de estudos comparativos, em maior profundidade, entre os principais modelos de distribuição pública de medicamentos essenciais, como o Programa "Aqui Tem Farmácia Popular", distribuição nas unidades básicas de saúde do SUS e programa "Remédio em Casa", em São Paulo, poderia servir de base para uma melhor definição das políticas públicas e estratégias de distribuição deles no Brasil.

Além dos custos de aquisição dos medicamentos, impostos e custos operacionais, poderiam ser incluídos outros aspectos nesta análise, como o custo da falta dos medicamentos, que pode aumentar os gastos com internações decorrentes das complicações na saúde da população devido à interrupção nos tratamentos; aumento das ações judiciais criadas para o custeio desses medicamentos que estão faltando; custos dos desperdícios pelas compras de remédios em excesso, com perdas dos produtos por prazos de validade vencidos e elevados custos de estoque; problemas de gestão, como a falta de capacitação de pessoal, falhas no planejamento de demanda dos remédios e uso de tecnologias de informação arcaicas no atendimento das unidades do SUS. Estes são exemplos de ineficiências na gestão pública que, além de representarem desperdícios de recursos, oferecem "oportunidades" para "desvios" e "práticas não éticas", que acabam onerando ainda mais os cofres públicos.

Em 2016, em decorrência dos problemas de natureza política por que passou a sociedade brasileira, aguardavam-se alterações no programa. No início do ano, devido a ajustes fiscais, considerados necessários para o reequilíbrio das contas públicas, o Governo Federal reduziu os descontos nos medicamentos comercializados no programa "Aqui Tem Farmácia Popular". Também, no primeiro semestre de 2017, foi anunciado, pelo Governo Temer, o fim do programa na rede de farmácias próprias do governo, em razão dos altos custos de implantação e manutenção dessas farmácias.

Após o impeachment da Presidente Dilma Roussef, já no Governo Temer, quando argumentos de controle dos gastos públicos parecem questionar a viabilidade do próprio SUS, ainda em junho de 2017, o futuro do programa permanecia sem definição quanto à continuidade como política social de Estado, mas parte da imprensa já anunciou o seu fim. É inegável, entretanto, que, se for extinto, o Programa Farmácia Popular deixará lacuna no atendimento de camadas vulneráveis da população, o que exigirá sua superação.

\section{REFERÊNCIAS}

Amorim, M. C. S., \& Perillo, E. B. F. (Orgs.). (2008). Para entender a saúde no Brasil 2. São Paulo, SP: Editora LCTE.

Associação Brasileira das Indústrias de Medicamentos Genéricos. (2014). Entrevista por à S. R. P. de Almeida. São Paulo, SP.

Associação Brasileira de Rede de Farmácias e Drogarias. (2014). São Paulo, SP. 
Boschetti, I., Behring E. R., Santos, S. M. M., \& Mioto, R. C. T. (Orgs.). (2010). Capitalismo em crise: Política social e direitos. São Paulo, SP: Editora Cortez.

Caderno de Economia \& Negócios (2015, Junho 26). Fóruns Estadão Saúde. O Estado de S. Paulo, H5.

Carraro, W. B. W. H. (2014). Desenvolvimento econômico do Brasl e o programa Aqui Tem Farmácia Popular: Limitantes e potencialidades (Tese de doutorado, Faculdade de Ciências Econômicas da Universidade Federal do Rio Grande do Sul). Recuperado de http://hdl.handle.net/10183/103962

Santos-Pinto, C. B., Costa, N. R., \& Osorio-de-Castro, C. G. S. (2011). Quem acessa o Programa Farmácia Popular do Brasil? Aspectos do fornecimento público de medicamentos. Ciência \& Saúde Coletiva, 16(6), 2963-2973. doi:10.1590/S141381232011000600034

Faleiros, V. P. (2009). A política social do estado capitalista. São Paulo, SP: Editora Cortez.

Federação Brasileira da Indústria Farmacêutica. (2008). Saúde e indústria farmacêutica em debate. São Paulo, SP: Editora Cubzac.

Fundação Oswaldo Cruz. (2014). Entrevista realizada com o Departamento de Gerência Técnica da Fiocruz. Rio de Janeiro, RJ.

Gazeta Mercantil. (2000). A ind ústria farmacêutica. Panorama Setorial, I.
Marsiglia, R. G. (2014). Entrevista realizada com o Departamento de Medicina Social da Faculdade de Ciências Médicas Santa Casa de São Paulo e do Programa de Estudos Pós-Graduados em Serviço Social da Pontifícia Universidade Católica. São Paulo, SP.

Ministério da Saúde. (2013). Entrevista com - Coordenador Geral de Gestão do Programa Farmácia Popular. Brasília, DF.

Ministério da Saúde. (2015). Portal da Saúde. Brasília, DF. Recuperado de http://189.28.128.178/sage/

Oliveira, F. (2004). Por que política? Palestra no Fórum da Sociedade Civil na Unctad, São Paulo, SP. Recuperado de http://www. ibase.br/userimages/francisco_de_oliveira_ port.pdf

Pereira, P. A. P. (2008). Política social: Temas \& questões. São Paulo, SP: Editora Cortez.

Picarelli, M. F. S. P., \& Aranha, M. I. (2001). Política de patentes em saúde humana. São Paulo, SP: Editora Atlas.

Programa Farmácia Popular. (2014). Coordenadora nos anos de 2006 e 2007. São Paulo, SP.

Sindicato da Indústria de Produtos Farmacêuticos no Estado de São Paulo. (2013). Entrevistas com presidente executivo do Sindicato da Indústria de Produtos Farmacêuticos no Estado de São Paulo. São PauIo, SP.

Sindicato da Indústria de Produtos Farmacêuticos no Estado de São Paulo. (2014). 
O setor farmacêutico brasileiro: Desafios e tendências.

Vieira, F. S. (2008). Qualificação dos serviços farmacêuticos no Brasil: Aspectos inconclusos da agenda do Sistema Único de Saúde. Revista Panamericana de Salud Pública, 24(2), 91-100. doi:10.1590/S102049892008000800003

World Health Organization. (2001). How to develop and implement a national drug policy, 1988 (2nd ed.). Geneva, Switzerland. Recuperado de http://apps.who.int/iris/bitstr eam/10665/42423/1/924154547X.pdf
World Health Organization. (2004). The world medicines situation. Geneva, Switzerland. Recuperado de http://www.searo.who.int/ LinkFiles/Reports_World_Medicines_Situation.pdf

World Health Organization. (2006). Measuring transparency in medicines registration, selection and procurement. Geneva, Switzerland. Recuperado de http://www.who.int/ medicines/areas/policy/goodgovernance/ Transparency4CountryStudy.pdf 